Pacific

Journal of

Mathematics

A PRIMARY OBSTRUCTION TO TOPOLOGICAL EMBEDDINGS

FOR MAPS BETWEEN GENERALIZED MANIFOLDS

Carlos Biasi, Janey Daccach, and Osamu Saeki

Volume $197 \quad$ No. 2

February 2001 


\title{
A PRIMARY OBSTRUCTION TO TOPOLOGICAL EMBEDDINGS

\author{
FOR MAPS BETWEEN GENERALIZED MANIFOLDS
}

\author{
Carlos Biasi, Janey Daccach, and Osamu Saeki
}

\begin{abstract}
For a proper continuous map $f: M \rightarrow N$ between smooth manifolds $M$ and $N$ with $m=\operatorname{dim} M<\operatorname{dim} N=m+k$, a homology class $\theta(f) \in H_{m-k}^{c}\left(M ; \mathbf{Z}_{2}\right)$ has been defined and studied by the first and the third authors, where $H_{*}^{c}$ denotes the singular homology with closed support. In this paper, we define $\theta(f)$ for maps between generalized manifolds. Then, using algebraic topological methods, we show that $\bar{f}_{*} \theta(f) \in$ $\check{H}_{m-k}^{c}\left(f(M) ; \mathrm{Z}_{2}\right)$ always vanishes, where $\bar{f}=f: M \rightarrow f(M)$ and $\check{H}_{*}^{c}$ denotes the Čech homology with closed support. As a corollary, we show that if $f$ is properly homotopic to a topological embedding, then $\theta(f)$ vanishes: In other words, the homology class can be regarded as a primary obstruction to topological embeddings. Furthermore, we give an application to the study of maps of the real projective plane into 3-dimensional generalized manifolds.
\end{abstract}

\section{Introduction.}

This paper is a continuation of the authors' studies [1], [2], [3], [4], and [5]. Let $M$ and $N$ be topological manifolds ${ }^{1}$ of dimensions $m$ and $n$ respectively and suppose $k=n-m>0$. For a proper continuous map $f: M \rightarrow N$, a homology class $\theta(f) \in H_{m-k}^{c}\left(M ; \mathbf{Z}_{2}\right)$ has been defined in [4, Definition 2.5], where $H_{*}^{c}$ denotes the singular homology of the compatible family with respect to compact subsets (see [21, Chapter 6, §3]), or equivalently the singular homology based on infinite chains (see $[\mathbf{1 9}, \S 5$ and $\S 65]$ ), or equivalently the singular homology with closed support (see [22, p. 118], [9]). This homology class is a proper homotopy invariant ${ }^{2}$ of $f$ and has the property that, when $M$ and $N$ are smooth manifolds, if $f$ is properly homotopic to a proper smooth embedding, then $\theta(f)$ vanishes. Furthermore, when $M$ is compact and $M$ and $N$ are smooth manifolds, if $f$

\footnotetext{
${ }^{1}$ In this paper, all manifolds have no boundary.

${ }^{2}$ Two proper continuous maps $f$ and $g: M \rightarrow N$ are properly homotopic if there exists a homotopy $F: M \times[0,1] \rightarrow N$ between $f$ and $g$ such that $F$ is a proper map.
} 
is homotopic to a topological embedding ${ }^{3}$, then $\theta(f)$ vanishes (see [4]). For all these results, the differentiable structures on both $M$ and $N$ have played an important role, since the technique of generic differentiable maps and the results due to Ronga [20] about such generic maps have been extensively used.

In this paper, we consider generalized manifolds and proper continuous maps between such spaces. Generalized manifolds of dimension $m$ which we consider in this paper are certain topological spaces $M$ such that for every $x \in M, H_{*}\left(M, M-\{x\} ; \mathbf{Z}_{2}\right)$ is isomorphic to $H_{*}\left(\mathbf{R}^{m}, \mathbf{R}^{m}-\{0\} ; \mathbf{Z}_{2}\right)$ (for details see §2). Note that, for such generalized manifolds, the Poincaré duality holds (see [6], [7], [8], [9]). Such manifolds have recently been studied extensively; for example, it has been known that there exist generalized manifolds which are not homotopy equivalent to topological manifolds (see $[\mathbf{1 0}],[11])$.

Let $f: M \rightarrow N$ be a proper continuous map between generalized manifolds with $m=\operatorname{dim} M<\operatorname{dim} N=m+k$. Since the definition of the homology class $\theta(f)$ for maps between topological manifolds depends only on the Poincaré duality, we can define the same class for maps between generalized manifolds as well. We then study the homology class $\theta(f)$ from an algebraic topological viewpoint, so that we need no differentiability hypothesis on $M$ or $N$. Our main result is Corollary 3.2, which states that $\bar{f}_{*} \theta(f) \in \check{H}_{m-k}^{c}\left(f(M) ; \mathbf{Z}_{2}\right)$ always vanishes, where $\bar{f}=f: M \rightarrow f(M)$ and $\check{H}_{*}^{c}$ denotes the Čech homology with closed support (for example, see [22, p. 146], [9]). This result implies, as a direct corollary, that if $f$ is a proper topological embedding, then $\theta(f) \in \check{H}_{m-k}^{c}\left(M ; \mathbf{Z}_{2}\right)$ vanishes (Corollary 3.5). This means that the homology class $\theta(f)$ can always be regarded as a primary obstruction to the existence of a proper homotopy between the given map $f$ and a proper topological embedding.

Using the above mentioned result, we give various related results as applications. First, as Corollary 3.9, we show that the top Stiefel-Whitney class of the stable normal bundle of a proper topological embedding, defined via the Stiefel-Whitney classes of the generalized manifolds involved, coincides with the modulo 2 normal Euler class of the embedding (see [12, Chapter VIII, $\S 11]$ ). Furthermore, we give a new proof of a result of Haefliger [15] (see also $[23])$ which states that the $i$-th Stiefel-Whitney class $w_{i}(f) \in H^{i}\left(M ; \mathbf{Z}_{2}\right)$ of the stable normal bundle of a codimension- $k$ proper topological embedding $f: M \rightarrow N$ vanishes for all $i>k$ (Corollary 3.10). Then we give an application concerning continuous maps of the real projective plane into 3dimensional generalized manifolds (Proposition 4.2), generalizing results of $[3]$.

${ }^{3} \mathrm{~A}$ continuous map is said to be a topological embedding if it is a homeomorphism onto its image. In particular, the topological embeddings in this paper may not necessarily be locally flat. 
We also give some new results about the homology class $\theta(f)$ itself. For example, we show that if the source generalized manifold $M$ is compact and the closure of the self-intersection set of $f$ has topological dimension strictly less than $m-k$, then $\theta(f) \in H_{m-k}\left(M ; \mathbf{Z}_{2}\right)$ vanishes (Corollary 3.17).

The paper is organized as follows. In $\S 2$, we give a precise definition of generalized manifolds and define the homology class $\theta(f)$. We will see that this class depends only on the map $f: M \rightarrow V$, where $V$ is an arbitrary open neighborhood of $f(M)$ in $N$. In $\S 3$, we show our key theorem (Theorem 3.1) and give various corollaries. Finally, in $\S 4$, we give an application to continuous maps of the real projective plane into 3-dimensional generalized manifolds (Proposition 4.2), which can be regarded as a refinement of a result obtained in $[\mathbf{3}]$.

Throughout the paper, homology and cohomology groups always have

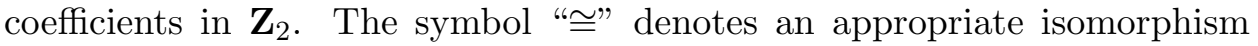
between algebraic objects.

\section{Preliminaries.}

In this section, we give precise definitions of generalized manifolds and the primary obstruction $\theta$.

Definition 2.1. A locally compact Hausdorff space $M$ is a generalized manifold of dimension $m$ if the following conditions are satisfied.

(1) $M$ is hereditarily paracompact (see Remark 2.2 (1) below).

(2) $\operatorname{dim}_{\mathbf{Z}_{2}} M<+\infty$ in the sense of [9] (see Remark 2.2 (2) below).

(3) $M$ is $H L C_{\mathbf{Z}_{2}}^{\infty}$ in the sense of [9, p. 35] (see Remark 2.2 (3) below).

(4) $H_{*}(M, M-\{x\}) \cong H_{*}\left(\mathbf{R}^{m}, \mathbf{R}^{m}-\{0\}\right)$ for every $x \in M$.

Remark 2.2. (1) A topological space is hereditarily paracompact if every open subset is paracompact (see $[\mathbf{9}$, Chapter $1, \S 6]$ ). This condition is necessary in order to guarantee that every open subset of a generalized manifold is again a generalized manifold.

(2) By $\left[\mathbf{9}, 16.14\right.$ Theorem, p. 115], $\operatorname{dim}_{\mathbf{Z}_{2}} M<+\infty$ if and only if there exists an $l<+\infty$ such that $H_{c}^{l+1}(U)=0$ for all open subset $U$ of $M$, where $H_{c}^{*}$ denotes the singular cohomology with compact support.

(3) A topological space $X$ is said to be $H L C_{\mathbf{Z}_{2}}^{\infty}$ if for each $x \in X$, each neighborhood $U$ of $x$ in $X$, and each $p$, there exists a neighborhood $V \subset U$ of $x$ such that the homomorphism $\tilde{H}_{p}(V) \rightarrow \tilde{H}_{p}(U)$ induced by the inclusion is trivial (see $\left[\mathbf{9}\right.$, p. 35]), where $\tilde{H}_{*}$ denotes the reduced singular homology. Note that this condition together with the condition (1) implies, in our situation, that the sheaf theoretic homologies and cohomologies coincide with the singular homologies and cohomologies respectively (see [9]).

(4) Recall that a topological space $X$ is said to be an ENR (Euclidean neighborhood retract) if there exist a subspace $Y$ of some $\mathbf{R}^{n}$ homeomorphic 
to $X$, a neighborhood $V$ of $Y$, and a retraction $r: V \rightarrow Y$. If $M$ is an ENR and satisfies the condition (4) of Definition 2.1, then $M$ is a generalized manifold of dimension $m$. We call such a space an ENR $\mathbf{Z}_{2}$-homology manifold. In particular, second countable topological manifolds are all ENR $\mathbf{Z}_{2}$-homology manifolds. Note that a separable metric space $X$ is an ENR if and only if it is a locally compact ANR (absolute neighborhood retract) and has finite topological dimension (see [16, Chapter V]). Note also that the homology manifolds constructed in [10], [11] are all ENR $\mathbf{Z}_{2}$-homology manifolds in the above sense ${ }^{4}$.

In $[6],[7]$, it has been shown that the Poincaré duality holds for locally orientable generalized manifolds. Furthermore, in [8], every generalized manifold has been shown to be locally orientable. Thus the Poincaré duality holds for all generalized manifolds (for details, see [9]). More precisely, if $M$ is a connected generalized manifold of dimension $m$, then $H_{m}^{c}(M)$ is isomorphic to $\mathbf{Z}_{2}$ and the homomorphisms

$$
\begin{array}{ll}
\frown[M] \quad: \quad H^{i}(M) \rightarrow H_{m-i}^{c}(M), \\
\frown[M]: \quad H_{c}^{i}(M) \rightarrow H_{m-i}(M)
\end{array}
$$

are isomorphisms for all $i$, where $[M]$ is the generator of $H_{m}^{c}(M)$ and is called the fundamental class of $M$ (see also Remark 2.3 below). We call the above isomorphisms the Poincaré duality isomorphisms and denote them by $D_{M}$.

Remark 2.3. Note that the universal coefficient theorem works for cohomology with compact support and homology with closed support. In fact, for a topological space $X$, the cup product $^{5}$ is a bilinear map

$$
\begin{array}{lll}
H^{i}(X) \times H^{j}(X) \rightarrow H^{i+j}(X) & \text { or } \\
H_{c}^{i}(X) \times H^{j}(X) \rightarrow H_{c}^{i+j}(X) & \text { or } \\
H_{c}^{i}(X) \times H_{c}^{j}(X) \rightarrow H_{c}^{i+j}(X), &
\end{array}
$$

the cap product is a bilinear map

$$
\begin{aligned}
& H^{i}(X) \times H_{j}(X) \rightarrow H_{j-i}(X) \text { or } \\
& H^{i}(X) \times H_{j}^{c}(X) \rightarrow H_{j-i}^{c}(X) \text { or } \\
& H_{c}^{i}(X) \times H_{j}^{c}(X) \rightarrow H_{j-i}(X),
\end{aligned}
$$

and the scalar product (or the Kronecker index) $\langle$,$\rangle is a nonsingular bilinear$ map

$$
\begin{aligned}
H_{c}^{i}(X) \times H_{i}^{c}(X) & \rightarrow \mathbf{Z}_{2} \text { or } \\
H^{i}(X) \times H_{i}(X) & \rightarrow \mathbf{Z}_{2} .
\end{aligned}
$$

\footnotetext{
${ }^{4} \operatorname{In}[\mathbf{1 0}],[\mathbf{1 1}]$, they use integral coefficients in a condition corresponding to Definition 2.1 (4). Thus their condition always implies ours.

${ }^{5}$ For details, see $[\mathbf{2 2}$, p. 117] or $[\mathbf{1 4}, \S 26]$.
} 
Remark 2.4. Let $M$ be a generalized manifold. Then, by using the Poincaré duality isomorphisms together with the Steenrod squaring operation, we can define the Wu classes of $M$ and hence the Stiefel-Whitney classes by virtue of the $\mathrm{Wu}$ formula (for details, see $[\mathbf{1 8}, \S 11]$ ).

Let $M$ and $N$ be generalized manifolds of dimensions $m$ and $n$ respectively such that $k=n-m>0$ and $f: M \rightarrow N$ a proper continuous map. Let us denote by $U_{f} \in H^{k}(N)$ the Poincaré dual of $f_{*}[M] \in H_{m}^{c}(N)$; in other words, $f_{*}[M]=U_{f} \frown[N]$. Note that $f_{*}[M] \in H_{m}^{c}(N)$ is well-defined, since $f$ is a proper map (see [22, p. 118]).

Let the total Stiefel-Whitney classes of $M$ and $N$ be denoted by $w(M) \in$ $H^{*}(M)$ and $w(N) \in H^{*}(N)$ respectively (see Remark 2.4) and let $\bar{w}(M) \in$ $H^{*}(M)$ denote the dual Stiefel-Whitney class of $M$; i.e., $\bar{w}(M)=w(M)^{-1}$. Define $w(f)=\left(f^{*} w(N)\right) \smile \bar{w}(M)$, which is called the total Stiefel-Whitney class of the stable normal bundle of $f$. We denote by $w_{k}(f) \in H^{k}(M)$ the degree $k$ term of $w(f)$, which is the $k$-th Stiefel-Whitney class of the stable normal bundle of $f$.

Definition 2.5. We define

$$
\theta(f)=\left(f^{*} U_{f}-w_{k}(f)\right) \frown[M] \in H_{m-k}^{c}(M) .
$$

Note that this is a proper homotopy invariant of $f$. We also note that when $M$ is compact, $\theta(f)$ is an element of the usual homology group $H_{m-k}(M)$, since we have $H_{m-k}(M) \cong H_{m-k}^{c}(M)$.

The above homology class has been originally defined in [4] and denoted by $\theta_{1}(f)$ for proper continuous maps between topological manifolds. In this paper, we use the notation $\theta(f)$ instead of $\theta_{1}(f)$, which will cause no confusion.

The reason why we use the homology class instead of the corresponding cohomology class is that when $M$ and $N$ are smooth manifolds, $\theta(f)$ coincides with the fundamental class carried by the closure of the self-intersection set of a generic map properly homotopic to $f$ (see [20], [4]).

Many important observations about $f^{*} U_{f}$ and $w_{k}(f) \in H^{k}(M)$ have been given in $[4, \S 2]$, although the objects considered there are maps between topological manifolds. For example, the following lemma has already been observed in [4] in such situations. Here we give a different proof which works also for our more general setting. Note that, by Remark 2.2 (1), every open subset of a generalized manifold is again a generalized manifold.

Lemma 2.6. Let $V$ be an open subset of $N$ which contains $f(M)$ and consider the map $f_{V}=f: M \rightarrow V(\subset N)$. Then $\theta(f)=\theta\left(f_{V}\right)$ in $H_{m-k}^{c}(M)$.

Proof. Let $i: V \rightarrow N$ denote the inclusion map and $i_{\sharp}: H_{c}^{*}(V) \rightarrow H_{c}^{*}(N)$ the natural homomorphism induced by $i$ (for example, see $[\mathbf{1 4}, \S 26]$ ). Furthermore, let $i_{\sharp}^{*}: H_{*}^{c}(N)=\operatorname{Hom}\left(H_{c}^{*}(N), \mathbf{Z}_{2}\right) \rightarrow \operatorname{Hom}\left(H_{c}^{*}(V), \mathbf{Z}_{2}\right)=H_{*}^{c}(V)$ 
denote the dual homomorphism of $i_{\sharp}$. Then we have the following commutative diagram:

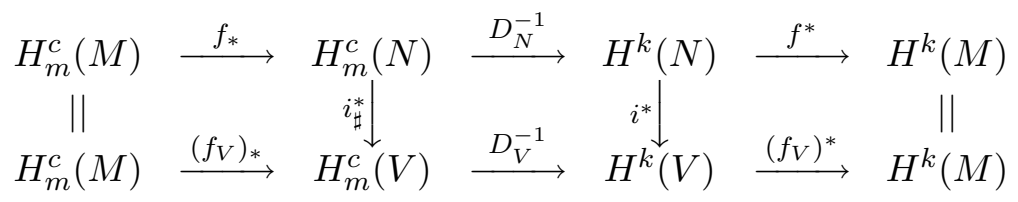

(for example, see $[\mathbf{1 4}, \S 26])$. Thus we see that

$$
f^{*} U_{f}=\left(f_{V}\right)^{*} U_{f_{V}}
$$

in $H^{k}(M)$.

Let $v$ denote the total $\mathrm{Wu}$ class. Then we have

$$
\langle x \smile v(N),[N]\rangle=\langle S q(x),[N]\rangle
$$

for all $x \in H_{c}^{*}(N)$. Let $y \in H_{c}^{*}(V)$ be an arbitrary element. Substituting $x=i_{\sharp} y$ in (2.3), we have

$$
\left\langle i_{\sharp} y \smile v(N),[N]\right\rangle=\left\langle S q\left(i_{\sharp} y\right),[N]\right\rangle .
$$

The left-hand side of (2.4) is equal to

$$
\left\langle i_{\sharp}\left(y \smile i^{*} v(N)\right),[N]\right\rangle=\left\langle y \smile i^{*} v(N),[V]\right\rangle,
$$

and the right-hand side of (2.4) is equal to

$$
\left\langle i_{\sharp} S q(y),[N]\right\rangle=\langle S q(y),[V]\rangle .
$$

Thus we have

$$
\left\langle y \smile i^{*} v(N),[V]\right\rangle=\langle S q(y),[V]\rangle
$$

for all $y \in H_{c}^{*}(V)$, which implies that $v(V)=i^{*} v(N)$. Then by Wu's formula, we have $w(V)=i^{*} w(N)$. This shows that

$$
w(f)=w\left(f_{V}\right) .
$$

Then Equations (2.2) and (2.8) together with Definition 2.5 show that $\theta(f)=\theta\left(f_{V}\right)$ in $H_{m-k}^{c}(M)$. This completes the proof.

It will be shown in the next section that if $f$ is properly homotopic to a proper topological embedding, then $\theta(f) \in \check{H}_{m-k}^{c}(M)$ vanishes. In other words, $\theta(f)$ can be regarded as a primary obstruction to the existence of such a proper homotopy. 


\section{Key theorem and corollaries.}

In this section, we first prove the following key theorem and give its important corollaries.

Theorem 3.1. Let $f: M \rightarrow N$ be a proper continuous map of an $m$ dimensional generalized manifold $M$ into an $(m+k)$-dimensional generalized manifold $N$ with $k>0$. Then $f_{*} \theta(f) \in H_{m-k}^{c}(N)$ always vanishes.

Proof. First recall that

$$
\begin{aligned}
f_{*} \theta(f) & =f_{*}\left(\left(f^{*} U_{f}-w_{k}(f)\right) \frown[M]\right) \\
& =f_{*}\left(\left(f^{*} U_{f}\right) \frown[M]\right)-f_{*}\left(w_{k}(f) \frown[M]\right),
\end{aligned}
$$

where $w_{k}(f)$ is equal to the degree $k$ term of $\left(f^{*} w(N)\right) \smile \bar{w}(M)$. As to the first term of Equation (3.2), we have

$$
\begin{aligned}
f_{*}\left(\left(f^{*} U_{f}\right) \frown[M]\right) & =U_{f} \frown f_{*}[M] \\
& =U_{f} \frown\left(U_{f} \frown[N]\right) \\
& =\left(U_{f} \smile U_{f}\right) \frown[N] .
\end{aligned}
$$

On the other hand, as to the second term of Equation (3.2), we have (3.6) $f_{*}\left(\left(\left(f^{*} w(N)\right) \smile \bar{w}(M)\right) \frown[M]\right)=f_{*}\left(\left(f^{*} w(N)\right) \frown(\bar{w}(M) \frown[M])\right)$.

Denoting by $\mathcal{D}$ the Steenrod squaring operation on the homology as defined in $[\mathbf{2 2}, \text { p. } 152]^{6}$, we have

$$
\bar{w}(M) \frown[M]=\mathcal{D}[M] .
$$

Thus, with the usual Steenrod squaring operation on the cohomology being denoted by $S q$, the right-hand side of Equation (3.6) is equal to

$$
\begin{aligned}
f_{*}\left(\left(f^{*} w(N)\right) \frown(\bar{w}(M) \frown[M])\right) & =f_{*}\left(\left(f^{*} w(N)\right) \frown \mathcal{D}[M]\right) \\
& =w(N) \frown f_{*} \mathcal{D}[M] \\
& =S q(v(N)) \mathcal{D}\left(f_{*}[M]\right) \\
& =\mathcal{D}\left(v(N) \frown f_{*}[M]\right) \\
& =\mathcal{D}\left(v(N) \frown\left(U_{f} \frown[N]\right)\right) \\
& =\mathcal{D}\left(\left(v(N) \smile U_{f}\right) \frown[N]\right),
\end{aligned}
$$

where Equation (3.10) follows from the $\mathrm{Wu}$ formula. Thus, we have only to show that $\left(U_{f} \smile U_{f}\right) \frown[N]$ is equal to the degree $m-k$ term of $\mathcal{D}\left(\left(v(N) \smile U_{f}\right) \frown[N]\right)$ in view of Equations (3.2), (3.5), (3.6) and (3.13).

\footnotetext{
${ }^{6}$ The operation $\mathcal{D}$ here corresponds to $\sum_{i} \mathcal{D}_{i}$ in the notation of [22]. Note also that $\mathcal{D}$ is also defined in [18, Problem 11-F, p. 136] and [21, Chapter 6, §10] and is denoted by $S q$ and $\overline{S q}$ respectively.
} 
Let $\xi$ be an arbitrary element of $H_{c}^{m-k}(N)$. By the universal coefficient theorem, we have only to show that

$$
\left\langle\xi,\left(U_{f} \smile U_{f}\right) \frown[N]\right\rangle=\left\langle\xi, \mathcal{D}\left(\left(v(N) \smile U_{f}\right) \frown[N]\right)\right\rangle .
$$

As to the left-hand side, we have

$$
\left\langle\xi,\left(U_{f} \smile U_{f}\right) \frown[N]\right\rangle=\left\langle\xi \smile\left(U_{f} \smile U_{f}\right),[N]\right\rangle .
$$

As to the right-hand side of Equation (3.14), we have

$$
\begin{aligned}
\left\langle\xi, \mathcal{D}\left(\left(v(N) \smile U_{f}\right) \frown[N]\right)\right\rangle & =\left\langle\overline{S q}(\xi),\left(v(N) \smile U_{f}\right) \frown[N]\right\rangle \\
& =\left\langle\left(\overline{S q}(\xi) \smile U_{f}\right) \smile v(N),[N]\right\rangle \\
& =\left\langle S q\left(\overline{S q}(\xi) \smile U_{f}\right),[N]\right\rangle \\
& =\left\langle S q(\overline{S q}(\xi)) \smile S q\left(U_{f}\right),[N]\right\rangle \\
& =\left\langle\xi \smile S q\left(U_{f}\right),[N]\right\rangle,
\end{aligned}
$$

where $\overline{S q}$ denotes the inverse of the automorphism $S q$ on the cohomology with compact support (see [18, Problem 11-E, p. 136]) $)^{7}$, Equation (3.16) follows from [18, Problem 11-F, p. 136] (or [22, (59)]), Equation (3.18) follows from the property of the Wu class, and Equation (3.20) follows from the definition of $\overline{S q}$. Since the degree $m+k$ term of $\xi \smile S q\left(U_{f}\right)$ is equal to the cup product of $\xi$ and the degree $2 k$ term of $S q\left(U_{f}\right)$, we see that

$$
\left\langle\xi \smile S q\left(U_{f}\right),[N]\right\rangle=\left\langle\xi \smile\left(U_{f} \smile U_{f}\right),[N]\right\rangle,
$$

which is equal to the left-hand side of Equation (3.14) by virtue of Equation (3.15), since $U_{f} \in H^{k}(N)$. This completes the proof.

Corollary 3.2. Let $f: M \rightarrow N$ be a proper continuous map of an $m$ dimensional generalized manifold $M$ into an $(m+k)$-dimensional generalized manifold $N$ with $k>0$. Then $\bar{f}_{*} \theta(f) \in \check{H}_{m-k}^{c}(f(M))$ always vanishes, where $\bar{f}=f: M \rightarrow f(M)$.

Remark 3.3. Note that there exists a natural homomorphism $H_{*}^{c}(M) \rightarrow$ $\check{H}_{*}^{c}(M)$. By $\theta(f)$, we mean either the homology class of $H_{m-k}^{c}(M)$ as defined in Definition 2.5 or its image in $\check{H}_{m-k}^{c}(M)$ by the above homomorphism. It is known that for ANR's, the Cech homology groups are naturally isomorphic to the singular homology groups: More precisely, the above homomorphism is an isomorphism (for example, see [21]). Furthermore, this is also true for compact generalized manifolds (see [9, 3.15, p. 298]). Thus, for such spaces, we always identify the two homology groups, especially for ENR $\mathbf{Z}_{2}$-homology manifolds and compact generalized manifolds.

\footnotetext{
${ }^{7}$ In the notation of $\left[\mathbf{2 2}\right.$, p. 161], we have $\overline{S q}=\sum_{i} Q_{i}$.
} 
Proof of Corollary 3.2. Take an arbitrary open neighborhood $V$ of $f(M)$ in $N$. Since $V$ is a generalized manifold, by applying Theorem 3.1 to $f_{V}=f$ : $M \rightarrow V$, we see that $\left(f_{V}\right)_{*} \theta\left(f_{V}\right)=0$ in $H_{m-k}^{c}(V)$. Since $\theta(f)=\theta\left(f_{V}\right)$ in $H_{m-k}^{c}(M)$ by Lemma 2.6, we see that $\left(f_{V}\right)_{*} \theta(f)=0$ in $H_{m-k}^{c}(V)$.

Let $i_{V}: f(M) \rightarrow V$ denote the inclusion map. Then, by the above argument, we have $\left(i_{V}\right)_{*}\left(\bar{f}_{*} \theta(f)\right)=\left(f_{V}\right)_{*} \theta(f)=0$ in $H_{m-k}^{c}(V)$ for all $V$. Since $\check{H}_{m-k}^{c}(f(M))$ is identified with the inverse limit

$$
\lim _{\longleftarrow} H_{m-k}^{c}(V)
$$

with $V$ ranging over all open neighborhoods of $f(M)$, we see that $\bar{f}_{*} \theta(f)$ vanishes in $\check{H}_{m-k}^{c}(f(M))$. This completes the proof.

We have the following immediate corollaries.

Corollary 3.4. Let $f: M \rightarrow N$ be a proper continuous map of an $m$ dimensional generalized manifold $M$ into an $(m+k)$-dimensional generalized manifold $N$ with $k>0$. If $\bar{f}_{*}: \check{H}_{m-k}^{c}(M) \rightarrow \check{H}_{m-k}^{c}(f(M))$ is a monomorphism, then $\theta(f)=0$ in $\check{H}_{m-k}^{c}(M)$.

Corollary 3.5. Let $f: M \rightarrow N$ be a proper topological embedding of an $m$ dimensional generalized manifold $M$ into an $(m+k)$-dimensional generalized manifold $N$ with $k>0$. Then $\theta(f) \in \check{H}_{m-k}^{c}(M)$ vanishes.

Remark 3.6. In Corollary 3.5, $\theta(f)$ vanishes as an element of $H_{m-k}^{c}(M)$ as well, provided that $M$ is compact or is an ANR (see Remark 3.3).

Corollary 3.5 shows that if a proper continuous map $f: M \rightarrow N$ between generalized manifolds $(\operatorname{dim} M<\operatorname{dim} N)$ is properly homotopic to a proper topological embedding, then $\theta(f)$ vanishes. In other words, $\theta(f)$ can be regarded as a primary obstruction to the existence of such a proper homotopy. This fact has already been shown in [4] when $M$ and $N$ are differentiable manifolds and $M$ is compact.

Remark 3.7. A topological embedding $f: M \rightarrow N$ between generalized manifolds is proper if and only if $f(M)$ is a closed subset of $N$.

Remark 3.8. Haefliger [15] has defined another type of an obstruction for maps between topological manifolds using a different method. In fact, using his obstruction, he has shown that, when $M$ and $N$ are compact topological manifolds, if $f: M \rightarrow N$ is a topological embedding, then $w_{i}(f)=0$ for all $i>k$ and that

$$
\left(w_{k}(f) \times 1\right) \smile \Delta_{M}^{*}=(f \times f)^{*} \Delta_{N}^{*} \in H^{n}(M \times M),
$$

where $1 \in H^{0}(M)$ is the identity element, and $\Delta_{M}^{*} \in H^{m}(M \times M)$ and $\Delta_{N}^{*} \in H^{n}(N \times N)$ are the Poincaré duals of the diagonals of $M \times M$ and $N \times N$ respectively (see $[\mathbf{1 5}, 5.2$ Théorème], [23]). It is not difficult to show 
that Equation (3.22) implies that our $\theta(f)$ vanishes. Thus our Corollary 3.5 can be regarded as a special case of Haefliger's result at least when $M$ and $N$ are compact topological manifolds.

Let $f: M \rightarrow N$ be a proper topological embedding as in Corollary 3.5 and suppose that $M$ and $N$ are ENR $\mathbf{Z}_{2}$-homology manifolds. Let us denote by $\tau(f) \in H^{k}(N, N-f(M))$ the Thom class (see [12, Chapter VIII, $\left.\S 11\right]^{8}$ ) and set $\chi(f)=f^{*} \tau(f) \in H^{k}(M)$, where $f^{*}: H^{k}(N, N-f(M)) \rightarrow H^{k}(M)$ is the homomorphism induced by the composition of $f: M \rightarrow N$ and the inclusion $N \rightarrow(N, N-f(M))$. The cohomology class $\chi(f)$ is called the normal Euler class of $f$ (see [12, Chapter VIII, §11]).

Corollary 3.9. Let $f: M \rightarrow N$ be a proper topological embedding of an $m$-dimensional ENR $\mathbf{Z}_{2}$-homology manifold $M$ into an $(m+k)$-dimensional ENR $\mathbf{Z}_{2}$-homology manifold $N$ with $k>0$. Then we have $w_{k}(f)=\chi(f)=$ $f^{*} U_{f} \in H^{k}(M)$.

Proof. By [12, Chapter VIII, 11.25 Proposition], we see that the element $\chi(f) \frown[M] \in H_{m-k}^{c}(M)$ is Poincaré dual to $f^{*} U_{f} \in H^{k}(M)$ (see also [12, Chapter VIII, §11.27]). Hence $\chi(f)=f^{*} U_{f}$. On the other hand, by Corollary 3.5 and the definition of $\theta(f)$ (see Definition 2.5), we have $w_{k}(f)=f^{*} U_{f}$. This completes the proof.

The following result has already been proved by Haefliger when $M$ and $N$ are compact topological manifolds (see Remark 3.8).

Corollary 3.10. Let $f: M \rightarrow N$ be a proper topological embedding of an $m$-dimensional ENR $\mathbf{Z}_{2}$-homology manifold $M$ into an $(m+k)$-dimensional ENR $\mathbf{Z}_{2}$-homology manifold $N$ with $k>0$. Then the $i$-th Stiefel-Whitney class $w_{i}(f) \in H^{i}(M)$ of the stable normal bundle of $f$ vanishes for all $i>k$.

Proof. Consider the composition

$$
\tilde{f}: M \stackrel{f}{\longrightarrow} N=N \times\{0\} \stackrel{\eta}{\longrightarrow} N \times \mathbf{R}^{i-k},
$$

where $\eta$ is the inclusion map. It is not difficult to see that $H_{m}^{c}\left(N \times \mathbf{R}^{i-k}\right)$ is naturally isomorphic to $H_{m-(i-k)}^{c}(N) \otimes H_{i-k}^{c}\left(\mathbf{R}^{i-k}\right) \cong H_{m-(i-k)}^{c}(N)$. Thus we see that $U_{\tilde{f}}=0 \in H^{i}\left(N \times \mathbf{R}^{i-k}\right)$ and hence that $\theta(\tilde{f})$ coincides with the Poincaré dual of $w_{i}(\tilde{f})$. On the other hand, since $\tilde{f}$ is a proper topological embedding, $\theta(\tilde{f})$ vanishes by Corollary 3.5 (see also Remark 3.6). Thus we have $w_{i}(\tilde{f})=0 \in H^{i}(M)$. Thus we have only to show the following.

Lemma 3.11. We have $w(\tilde{f})=w(f) \in H^{*}(M)$.

\footnotetext{
${ }^{8}$ In [12], $M$ and $N$ are assumed to be topological manifolds; however, similar argument works for generalized manifolds as well. For example, imitate the argument in [14].
} 
Proof. We have only to show that $w(N) \in H^{*}(N)$ corresponds to $w(N \times$ $\left.\mathbf{R}^{i-k}\right) \in H^{*}\left(N \times \mathbf{R}^{i-k}\right)$ by the natural isomorphism $H^{*}(N) \cong H^{*}(N \times$ $\left.\mathbf{R}^{i-k}\right)$. By the $\mathrm{Wu}$ formula, this reduces to showing that $v(N)$ corresponds to $v\left(N \times \mathbf{R}^{i-k}\right)$, where $v$ denotes the total Wu class. For this, it suffices to show that, for an arbitrary element $x \in H_{c}^{n+(i-k)-j}\left(N \times \mathbf{R}^{i-k}\right)$, we have

$$
\left\langle x \smile v_{j}^{\prime}(N),\left[N \times \mathbf{R}^{i-k}\right]\right\rangle=\left\langle S q^{j}(x),\left[N \times \mathbf{R}^{i-k}\right]\right\rangle,
$$

where $v_{j}^{\prime}(N) \in H^{j}\left(N \times \mathbf{R}^{i-k}\right)$ is the element which corresponds to $v_{j}(N) \in$ $H^{j}(N)$. Since $H_{c}^{n+(i-k)-j}\left(N \times \mathbf{R}^{i-k}\right) \cong H_{c}^{n-j}(N) \otimes H_{c}^{i-k}\left(\mathbf{R}^{i-k}\right)$, we may assume that $x=x^{\prime} \times \zeta$ for some $x^{\prime} \in H_{c}^{n-j}(N)$, where $\zeta \in H_{c}^{i-k}\left(\mathbf{R}^{i-k}\right) \cong \mathbf{Z}_{2}$ is the generator. Then, with the generator of $H^{0}\left(\mathbf{R}^{i-k}\right) \cong \mathbf{Z}_{2}$ being denoted by 1 , we have

$$
\begin{aligned}
\left\langle x \smile v_{j}^{\prime}(N),\left[N \times \mathbf{R}^{i-k}\right]\right\rangle & =\left\langle\left(x^{\prime} \times \zeta\right) \smile\left(v_{j}(N) \times 1\right),[N] \times\left[\mathbf{R}^{i-k}\right]\right\rangle \\
& =\left\langle x^{\prime} \smile v_{j}(N),[N]\right\rangle \\
& =\left\langle S q^{j}\left(x^{\prime}\right),[N]\right\rangle \\
& =\left\langle S q^{j}\left(x^{\prime}\right) \times \zeta,[N] \times\left[\mathbf{R}^{i-k}\right]\right\rangle \\
& =\left\langle S q^{j}(x),\left[N \times \mathbf{R}^{i-k}\right]\right\rangle .
\end{aligned}
$$

This completes the proof of Lemma 3.11 and hence Corollary 3.10 as well.

Remark 3.12. The above lemma follows also from [22, Théorème III.10].

Remark 3.13. A result similar to Corollary 3.10 holds also for topological immersions (i.e., locally injective continuous maps) between topological manifolds as well. For details, see $[\mathbf{1 5}, \S 5.4]$.

Corollaries 3.5 and 3.10 imply, for example, that if the dual StiefelWhitney class $\bar{w}_{i}(M)$ of an $m$-dimensional ENR $\mathbf{Z}_{2}$-homology manifold $M$ does not vanish, then for all $j \leq i, M$ cannot be topologically embedded in $\mathbf{R}^{m+j}$ as a closed subset. This is a very strong result, since we are considering topological embeddings which are not necessarily locally flat even when $M$ is a topological manifold.

Now we give some results concerning the properties of the homology class $\theta(f)$ itself.

Corollary 3.14. Let $f: M \rightarrow N$ be a proper continuous map of a connected $m$-dimensional generalized manifold $M$ into a $2 m$-dimensional generalized manifold $N$. Then $\theta(f) \in H_{0}^{c}(M)$ always vanishes.

Proof. Since $\bar{f}_{*}: H_{0}^{c}(M) \rightarrow \check{H}_{0}^{c}(f(M))$ is always a monomorphism, we have the result by Corollary 3.4. 
For a continuous map $f: M \rightarrow N$ between generalized manifolds, we set

$$
M(f)=\left\{x \in M: f^{-1}(f(x)) \neq\{x\}\right\},
$$

which is called the self-intersection set of $f$ (see [20], [4]).

In the following results, the source manifold $M$ will be compact, since we will use the exactness of Čech homology for compact pairs (see [17] and [13, Chapter IX, §7]). In such cases, the homology class $\theta(f)$ will always be an element of the usual homology group $H_{m-k}(M)$, which is naturally isomorphic to $H_{m-k}^{c}(M)$. We also note that $\check{H}_{*}^{c}$ is isomorphic to $\check{H}_{*}$ for compact spaces, where $\check{H}_{*}$ denotes the (usual) Cech homology.

The following is a refinement of [4, Theorem 6.1].

Corollary 3.15. Let $f: M \rightarrow N$ be a continuous map of an $m$-dimensional compact generalized manifold $M$ into an $(m+k)$-dimensional generalized manifold $N$ with $k>0$. Set $A=\overline{M(f)}$ and $B=f(A)$. Then there exists an element $\mu \in \check{H}_{m-k}(A)$ such that

$$
j_{*} \mu=\theta(f) \in \check{H}_{m-k}(M)=H_{m-k}(M) \text { and }(f \mid A)_{*} \mu=0 \in \check{H}_{m-k}(B),
$$

where $j: A \rightarrow M$ is the inclusion map (when $A=\emptyset$, we regard $\check{H}_{m-k}(A)=$ $\left.0=\check{H}_{m-k}(B)\right)$.

Proof. We may assume that $A \neq \emptyset$ by Corollary 3.5. By an argument as in $[5, \S 3]$, we have the following exact sequence:

$$
\check{H}_{m-k}(A) \stackrel{\alpha}{\longrightarrow} \check{H}_{m-k}(M) \oplus \check{H}_{m-k}(B) \stackrel{\psi}{\longrightarrow} \check{H}_{m-k}(f(M)),
$$

where $\alpha=\left(j_{*},(f \mid A)_{*}\right)$ and $\psi=\bar{f}_{*}+j_{*}^{\prime}$ with $j^{\prime}: B \rightarrow f(M)$ being the inclusion map. Since $\psi(\theta(f), 0)=0$ by Theorem 3.1, there exists an element $\mu \in \check{H}_{m-k}(A)$ such that $\alpha(\mu)=\left(j_{*} \mu,(f \mid A)_{*} \mu\right)=(\theta(f), 0)$. This completes the proof.

Corollary 3.15 shows that the "support" of the homology class $\theta(f) \in$ $H_{m-k}(M)$ is contained in the closure $A$ of the self-intersection set $M(f)$ of $f$. We do not know if $\mu \neq 0 \in \check{H}_{m-k}(A)$ when $A \neq \emptyset$. Note that, when $M$ and $N$ are smooth manifolds and $f$ is a generic map in the sense of Ronga [20], then the homology class $\mu \in \check{H}_{m-k}(A)$ can be chosen as the fundamental class carried by $A$ and then $\mu \neq 0$ whenever $A \neq \emptyset$, as has been shown in $[20]$.

Remark 3.16. By the same argument, we can show that if $v \in \check{H}_{m-k}(B)$ satisfies $j_{*}^{\prime} v=0$ in $\check{H}_{m-k}(f(M))$, then there exists an element $\mu_{v} \in \check{H}_{m-k}(A)$ such that $j_{*} \mu_{v}=\theta(f)$ and $(f \mid A)_{*} \mu_{v}=v$.

Corollary 3.17. Let $f: M \rightarrow N$ be a continuous map of an m-dimensional compact generalized manifold $M$ into an $(m+k)$-dimensional generalized manifold $N$ with $k>0$. Set $A=\overline{M(f)}$. If the topological dimension of $A$ is strictly less than $m-k$, then $\theta(f) \in H_{m-k}(M)$ vanishes. 
For the definition and the properties of the topological dimension, see [16].

Proof of Corollary 3.17. Since the topological dimension of $A$ is strictly less than $m-k$, we have $\check{H}_{m-k}(A)=0$ by [16, Theorem VIII 4 (p. 152)]. Then the result follows directly from Corollary 3.15.

\section{Maps of the projective plane into 3-dimensional generalized manifolds.}

In this section, we give an application of the results obtained in $\S 3$ to maps of the real projective plane into 3 -dimensional generalized manifolds.

Lemma 4.1. Let $f: \mathbf{R} P^{2} \rightarrow X$ be a continuous map of the real projective plane into a topological space $X$. If $f_{*}\left[\mathbf{R} P^{2}\right]=0$ in $H_{2}(X)$, then $f_{*}$ : $H_{1}\left(\mathbf{R} P^{2}\right) \rightarrow H_{1}(X)$ is the zero map.

Proof. Suppose that $f_{*}: H_{1}\left(\mathbf{R} P^{2}\right) \rightarrow H_{1}(X)$ is not the zero map. Since $H_{1}\left(\mathbf{R} P^{2}\right)$ is isomorphic to $\mathbf{Z}_{2}, f_{*}: H_{1}\left(\mathbf{R} P^{2}\right) \rightarrow H_{1}(X)$ must be injective. Then, by the universal coefficient theorem, $f^{*}: H^{1}(X) \rightarrow H^{1}\left(\mathbf{R} P^{2}\right)$ is surjective. Thus, there exists an element $\xi \in H^{1}(X)$ such that $f^{*} \xi \in$ $H^{1}\left(\mathbf{R} P^{2}\right) \cong \mathbf{Z}_{2}$ is the generator. Since $\left(f^{*} \xi\right) \smile\left(f^{*} \xi\right)=f^{*}(\xi \smile \xi)$ is the generator of $H^{2}\left(\mathbf{R} P^{2}\right) \cong \mathbf{Z}_{2}$, we see that $f^{*}: H^{2}(X) \rightarrow H^{2}\left(\mathbf{R} P^{2}\right)$ is surjective. Thus, again by the universal coefficient theorem, $f_{*}: H_{2}\left(\mathbf{R} P^{2}\right) \rightarrow$ $H_{2}(X)$ is injective. This contradicts our assumption. Thus $f_{*}: H_{1}\left(\mathbf{R} P^{2}\right) \rightarrow$ $H_{1}(X)$ must be the zero map. This completes the proof.

Proposition 4.2. Let $f: \mathbf{R} P^{2} \rightarrow N$ be a continuous map of the real projective plane into a 3-dimensional generalized manifold $N$. If $f_{*}\left[\mathbf{R} P^{2}\right]=0$ in $H_{2}(N)$, then $\bar{f}_{*}: H_{1}\left(\mathbf{R} P^{2}\right) \rightarrow \check{H}_{1}\left(f\left(\mathbf{R} P^{2}\right)\right)$ is the zero map.

Proof. By Lemma 4.1, $f_{*}: H_{1}\left(\mathbf{R} P^{2}\right) \rightarrow H_{1}(N)$ is the zero map. Hence $f$ is homologous to a constant map $g: \mathbf{R} P^{2} \rightarrow N$; i.e., $f_{*}=g_{*}: H_{*}\left(\mathbf{R} P^{2}\right) \rightarrow$ $H_{*}(N)$. As the definition of the primary obstruction (see Definition 2.5) shows, $\theta(f)$ is a homology invariant; i.e., if $f_{*}=g_{*}$, then $\theta(f)=\theta(g)$. Thus we have

$$
\theta(f)=\bar{w}_{1}\left(\mathbf{R} P^{2}\right) \frown\left[\mathbf{R} P^{2}\right],
$$

which is the generator of $H_{1}\left(\mathbf{R} P^{2}\right) \cong \mathbf{Z}_{2}$. Then the result follows from Corollary 3.2. This completes the proof.

Remark 4.3. When $N$ is a topological manifold, we can also prove the above proposition by using the fact that $f$ is bordant to a constant map and by using a result in [3]. Here we have given a proof which does not depend on the argument or the result in $[3]$.

As a direct corollary to the above proposition, we obtain the following. 
Corollary 4.4. Let $f: \mathbf{R} P^{2} \rightarrow N$ be a continuous map of the real projective plane into a 3-dimensional generalized manifold $N$. If $f_{*}\left[\mathbf{R} P^{2}\right]=0$ in $H_{2}(N)$, then $f$ is not a topological embedding.

Compare the above result with [3, Corollary 4.2].

Remark 4.5. A 2-dimensional compact generalized manifold is said to be a cohomology real projective plane if it has the same cohomology ring over $\mathbf{Z}_{2}$ as $\mathbf{R} P^{2}$. The results of this section hold also for maps of cohomology real projective planes into 3-dimensional generalized manifolds. Note, however, that if a cohomology real projective plane is second countable ${ }^{9}$, then it is homeomorphic to $\mathbf{R} P^{2}$ (see [9, 16.32 Theorem, p. 388]).

\section{References}

[1] C. Biasi, J. Daccach and O. Saeki, On R-bordism of maps and obstruction to topological embeddings, to appear in Osaka J. Math.

[2] C. Biasi and O. Saeki, On the Betti number of the image of a codimension- $k$ immersion with normal crossings, Proc. Amer. Math. Soc., 123 (1995), 3549-3554.

[3] _ On bordism invariance of an obstruction to topological embeddings, Osaka J. Math., 33 (1996), 729-735.

[4] - On the self-intersection set and the image of a generic map, Math. Scand., 80 (1997), 5-24.

[5] - On the Betti number of the image of a generic map, Comment. Math. Helv., 72 (1997), 72-83.

[6] A. Borel, The Poincaré duality in generalized manifolds, Mich. Math. J., 4 (1957), 227-239.

[7] A. Borel et al., Seminar on Transformation Groups, Ann. of Math. Studies, Vol. 46, Princeton Univ. Press, Princeton, N. J., 1960.

[8] G.E. Bredon, Wilder manifolds are locally orientable, Proc. Nat. Acad. Sci. U.S.A., 63 (1969), 1079-1081.

[9] _ Sheaf Theory, second edition, Graduate Texts in Math., Vol. 170, SpringerVerlag, New York, 1997.

[10] J. Bryant, S. Ferry, W. Mio and S. Weinberger, Topology of homology manifolds, Bull. Amer. Math. Soc., 28 (1993), 324-328.

[11] _ Topology of homology manifolds, Ann. of Math., 143 (1996), 435-467.

[12] A. Dold, Lectures on Algebraic Topology, Die Grundlehren der math. Wissenschaften, Band 200, Springer-Verlag, Berlin, Heidelberg, New York, 1972.

[13] S. Eilenberg and N. Steenrod, Foundations of Algebraic Topology, Princeton Univ. Press, Princeton, N. J., 1952.

[14] M.J. Greenberg, Lectures on Algebraic Topology, W.A. Benjamin, Inc., New York, Amsterdam, 1967.

[15] A. Haefliger, Points multiples d'une application et produit cyclique reduit, Amer. J. Math., 83 (1961), 57-70.

\footnotetext{
${ }^{9}$ This condition is equivalent to that it is metrizable.
} 
[16] W. Hurewicz and H. Wallman, Dimension Theory, Princeton Math. Series, Vol. 4, Princeton Univ. Press, Princeton, 1948.

[17] G.M. Kelly, The exactness of Čech homology over a vector space, Proc. Camb. Phil. Soc., 57 (1961), 428-429.

[18] J. Milnor and J. Stasheff, Characteristic Classes, Ann. of Math. Studies, Vol. 76, Princeton Univ. Press, Princeton, N. J., 1974.

[19] J.R. Munkres, Elements of Algebraic Topology, Addison-Wesley Publ. Company, California, Massachusetts, London, Amsterdam, Ontario, Sydney, 1984.

[20] F. Ronga, 'La classe duale aux points doubles' d'une application, Compositio Math., 27 (1973), 223-232.

[21] E.H. Spanier, Algebraic Topology, TATA McGraw-Hill Publ. Company Ltd., Bombay, New Delhi, 1966.

[22] R. Thom, Espaces fibrés en sphère et carrés de Steenrod, Ann. Sci. Ecole Norm. Sup., 69 (1952), 109-181.

[23] T. Yasui, On the Stiefel-Whitney classes of maps and Haefliger's obstructions to embeddings, Mem. Fac. Sci. Kochi Univ. Ser. A Math., 19 (1998), 73-84.

Received December 21, 1998.

Universidade de São Paulo

13560-970, SÃo CARlos, SP

BRAZIL

E-mail address: biasi@icmc.sc.usp.br

Universidade Estadual De Maringá

87020-900, MARINGÁ, PR

BRAZIL

E-mail address: janey@gauss.dma.uem.br

Hiroshima UNIVERSITY

Higashi-Hiroshima 739-8526

JAPAN

E-mail address: saeki@math.sci.hiroshima-u.ac.jp 